\title{
trans-Dominant Inhibition of Geminiviral DNA Replication by Bean Golden Mosaic Geminivirus rep Gene Mutants
}

\author{
Stephen F. Hanson and Douglas P. Maxwell
}

Department of Plant Pathology, University of Wisconsin, Madison 53706.

Accepted for publication 4 February 1999.

\begin{abstract}
Hanson, S. F., and Maxwell, D. P. 1999. trans-Dominant inhibition of geminiviral DNA replication by bean golden mosaic geminivirus rep gene mutants. Phytopathology 89:480-486.

Geminiviruses are a group of single-stranded DNA viruses that cause major losses on a number of important crops throughout the world. Bean golden mosaic virus (BGMV) is a typical bipartite, whitefly-transmitted geminivirus that causes a severe disease on beans (Phaseolus vulgaris) in the Western Hemisphere. The lack of natural resistance to geminiviruses has led to attempts to engineer resistance, particularly through the use of pathogen-derived resistance strategies. The rep gene contains several conserved domains including nucleoside triphosphate (NTP)-binding and DNA-nicking domains and is the only geminiviral gene necessary for

replication. Previous analysis by our group and others has demonstrated that the NTP-binding and DNA-nicking domains are necessary for geminiviral DNA replication. The ability of the rep gene and rep gene mutants to interfere with geminiviral DNA replication, when expressed in trans, was examined using a transient assay in a tobacco suspension cell culture system. Wild-type (wt) and mutant rep genes were cloned into plasmids under the control of the cauliflower mosaic virus $35 \mathrm{~S}$ promoter for in planta expression and coinoculated into tobacco cells with infectious clones of various geminiviruses. The wt rep gene from BGMV-GA was able to support replication of BGMV-GA DNA-B. Several different rep gene mutants, with function-abolishing mutations in the NTP-binding or DNA-nicking domains, were potent trans-dominant inhibitors of geminiviral DNA replication.
\end{abstract}

Geminiviruses are single-stranded DNA, plant-infecting viruses that cause severe disease problems on crops throughout the world $(7,21,24,45)$. In many areas, geminiviruses are the greatest constraint on production of important crops such as cassava, beans, mung beans, peppers, tomatoes, and cotton $(6,7,21)$. In the continental United States, geminiviruses cause severe problems on tomatoes, peppers, melons, and beans $(1,4,7)$. Bean golden mosaic virus (BGMV) is a typical bipartite, whitefly-transmitted geminivirus, is the largest constraint on bean production in Latin America (13), and causes substantial losses on pole beans in Florida (4).

Geminiviruses replicate via a rolling-circle mechanism $(37,40)$ similar to bacteriophages such as $\Phi \times 174$ (46). The rep gene encodes a complex, multifunctional protein that acts as a rolling-circle initiator protein, analogous to protein A of $\Phi \mathrm{X} 174$ (46), and is the only geminiviral gene necessary for replication $(11,12,22)$. The ac3 gene is not necessary for replication, but increases the efficiency of Rep protein-directed replication $(33,36,44)$. We previously demonstrated that the conserved DNA-nicking (27) and nucleoside triphosphate (NTP)-binding (23) domains within the rep gene are necessary for replication of the Guatemalan isolate of bean golden mosaic virus (BGMV-GA). Other researchers have reported similar results for other geminiviruses including tomato yellow leaf curl virus (TYLCV) $(8,29)$ and tomato golden mosaic virus (TGMV) (10). Biochemical analysis of the Rep protein of TYLCV-Sar has demonstrated that these domains have the predicted NTP-hydrolyzing and DNA-nicking activities in vitro $(8,28,29)$. In addition

Corresponding author: S. F. Hanson; E-mail address: sfhanson@facstaff.wisc.edu

Nucleotide and amino acid sequence data can be found in GenBank as accession no. M91604.

Publication no. P-1999-0322-01R

This article is in the public domain and not copyrightable. It may be freely reprinted with customary crediting of the source. The American Phytopathological Society, 1999. to its role in replication, the rep gene is also autoregulatory, controlling its expression at the level of transcription (43).

The origin of replication (ori) contains a series of direct repeat sequences located between the transcription and translation start sites for the rep gene $(10,13,14)$. TGMV and BGMV-GA Rep proteins bind to these direct repeat sequences (16), and this interaction is necessary for replication and autoregulation of the rep gene $(10,15)$. The direct repeats are generally conserved in position but vary in sequence between different phylogenetic clusters of geminiviruses $(2,13)$. The interaction between the Rep protein and the ori is highly specific, as demonstrated by the ability of Rep proteins from BGMV-GA and TGMV to interact with their cognate ori sequences but not to heterologous ori sequences (14). The ori also contains a sequence that can form a stem-loop structure that is almost perfectly conserved among all geminiviruses $(2,13)$. Rollingcircle replication is initiated by Rep protein-mediated cleavage in the loop sequence $(29,39)$.

Efforts to develop geminivirus-resistant beans and tomatoes have been underway for about 2 decades. These breeding programs have, in general, been unsuccessful $(3,32)$. The tyl gene, which confers partial resistance to tomato yellow leaf curl virus (TYLCV-IL), has been identified in wild tomato species $(34,48)$. Unfortunately, the tyl gene is not effective against other tomato-infecting geminiviruses such as tomato mottle virus (ToMoV) and has been difficult to introgress into commercial cultivars (J. T. Stout, personal communication). Another source of resistance to TYLCV, which has not been tested against other tomato-infecting geminiviruses, was recently introgressed into tomato from Lycopersicum hirsutum (47). A source of partial resistance to type II BGMV (13), a recessive gene ( $\mathrm{bgml}$ ) from breeding line A429 has been identified for beans (5) and has recently been incorporated into pole beans from Florida and small red dry beans from Honduras (J. S. Beaver, personal communication). It is not known if the bgml gene will confer resistance to other bean-infecting geminiviruses such as BGMV-BZ (Brazilian BGMV, type I isolate), bean dwarf mosaic virus (BDMV), or bean calico mosaic virus. 
The lack of natural resistance sources sufficient for control of geminiviral diseases has led to intensive efforts directed at engineering geminivirus resistance, particularly through the use of virusderived resistance schemes (17). The rep gene is an attractive target for engineering geminivirus resistance, since it is the only gene necessary for geminiviral DNA replication (22). We have proposed that mutations, which abolish the activity of the rep gene, may create trans-dominant rep genes $(23,27)$. Several examples of trans-dominant NTP-binding domain mutants of viral replicationassociated proteins have been described in animal virus systems $(30,38,42)$. The NTP-binding and DNA-nicking domains are attractive targets for mutation to engineer trans-dominant rep genes, since these motifs are conserved in all geminivirus rep genes and necessary for rep gene function.

Here we report on the effects of trans-expressed rep genes (wildtype [wt] and mutant rep) from BGMV-GA on replication of several bipartite geminiviruses in a tobacco suspension cell culture system. Our results show that nonfunctional rep mutants are trans-dominant inhibitors of replication for all geminiviruses tested and indicate that a trans-dominant interference strategy may be useful for engineering plants with broad-spectrum geminivirus resistance.

\section{MATERIALS AND METHODS}

Cloned geminiviral DNAs. Previously described infectious geminivirus clones were used in all experiments: pGAA2 (23) and pGAB1 (18) for BGMV-GA DNA-A and DNA-B, respectively; pBZA1 for BGMV-BZ DNA-A (18); pDRA1 for BGMV-DR (Dominican Republic BGMV, type II isolate) (18); and pBDA1 for BDMV DNA-A (18). All DNAs were excised from plasmid vectors (SpeI for pGAA2, BamHI for pGAB1, HindIII for pBZA1, and $B g l I I$ for pBDA1) and inoculated as linear unit-length genomic components. DNAs were extracted with phenol/chloroform/isoamyl alcohol $(25: 24: 1)$ and ethanol precipitated after digestion. DNA concentrations were determined using a fluorometer (Hoefer TKO100; Hoefer Scientific Instruments, San Francisco, CA) and adjusted to $1 \mu \mathrm{g} / \mu \mathrm{l}$ for inoculation by electroporation (23).
Construction of rep gene trans-expression vectors. Vectors constructed for the transient expression of the NTP-binding and DNA-nicking domain mutants of rep gene in plant cells were derived from the rep expression vector pWRG2288 (gift from D. R. Russell, Agracetus Inc.). This plasmid contains the rep gene of BGMV-GA cloned downstream of the cauliflower mosaic virus (CaMV) 35S promoter and an alfalfa mosaic virus leader sequence. An EcoRI/XhoI fragment containing the 35S-rep gene cassette was moved into pBSII-KS+ (pBS; Stratagene Inc., La Jolla, CA) creating the rep gene expression vector pTrep (Fig. 1). The pTrep plasmid codes for the entire rep gene, the n-terminal $60 \%$ (105 codons) of the trap gene, and the first 16 codons of the ac3 gene. Sitedirected mutagenesis was used to engineer a NcoI site in the start codon of the rep gene in pTrep (making pTrepN) to facilitate cloning of the DNA-nicking domain mutants. This mutation (CCATGC to CCATGg) changes the second codon of the rep protein from proline to alanine. This change was also made in wt DNA-A (pGAA2N) and had no detectable effect on replication in NT-1 (Nicotiana tabacum) tobacco suspension cells or infectivity of bean plants (data not shown). Rep trans-expression vectors containing the entire $a c 3$ gene were constructed by cloning of an EcoRI/XbaI fragment from pR2N (S. F. Hanson and D. P. Maxwell, unpublished data), which contains a rep/trap/ac3 cassette with an engineered $X b a \mathrm{I}$ site at nucleotide position 1,041, into pTrep and pTrepN, making pTrep23 and pTrep23N, respectively. Expression vectors containing the entire rep gene and a truncated $a c 3$ gene were made by self-ligation of pTrep23 and pTrep $23 \mathrm{~N}$, which had been digested with EcoRI ${ }^{1472}$ and $C l a I^{1299}$, followed by treatment with T4 DNA polymerase to blunt the incompatible ends. DNA sequence analysis showed that all clones had an in frame deletion in the ac 3 gene that removed codons 17 to 83 and inserted two nonsense codons at codon positions 17 and 18 in all pTrep $23 \Delta \mathrm{E} / \mathrm{C}$ clones.

NTP-binding domain mutations were introduced into Rep expression plasmids by cloning a $B g l \mathrm{II} / E c o$ RI fragment from mutant DNA-As (23) into pTrep in place of the wt BglII/EcoRI fragment (Fig. 1). DNAs containing DNA-nicking domain mutations were amplified by polymerase chain reaction (PCR) from mutant DNA-As
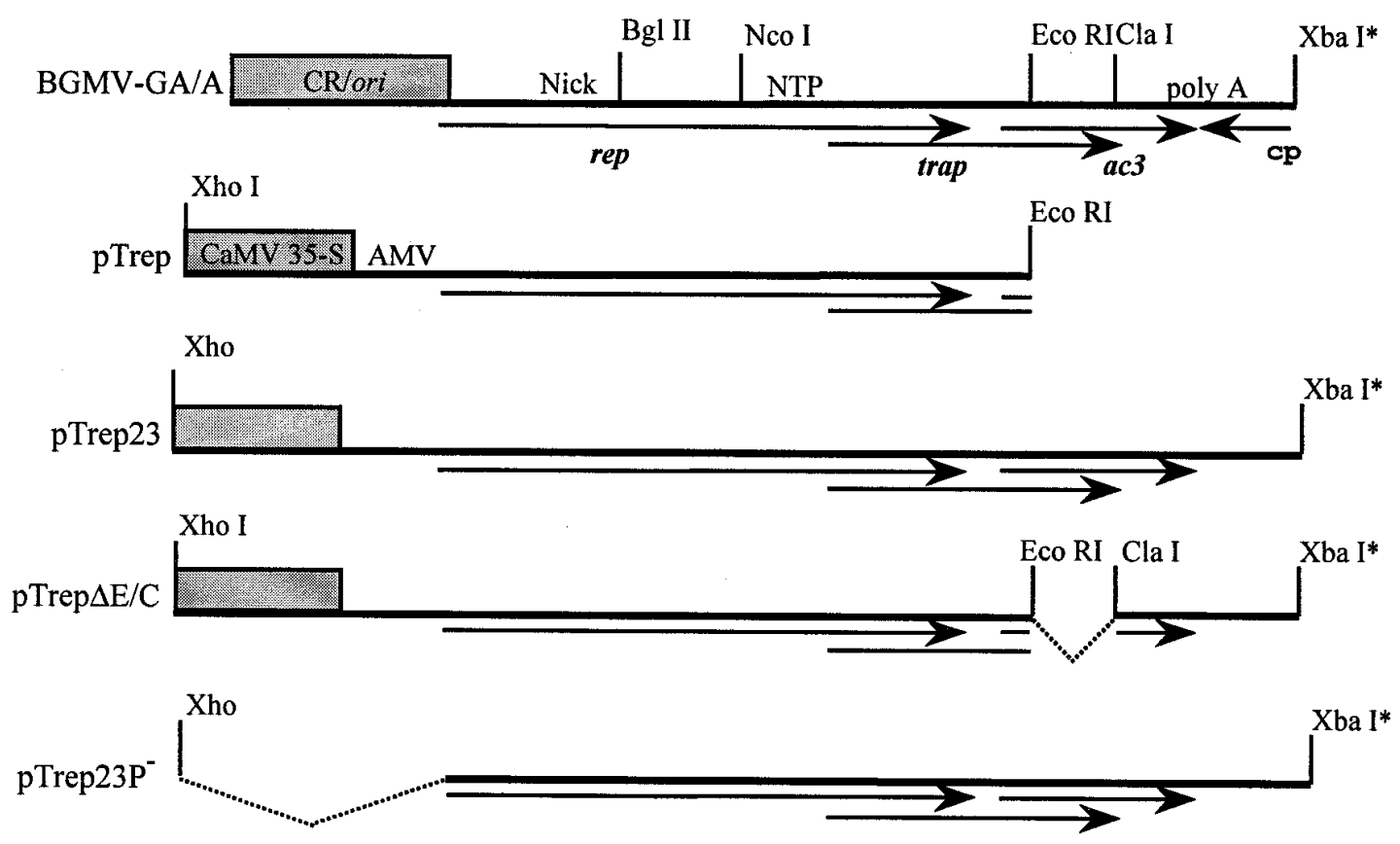

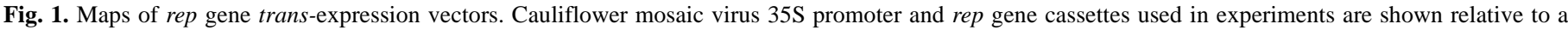

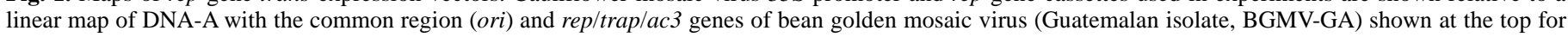

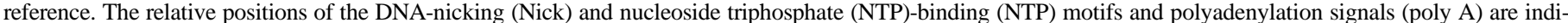

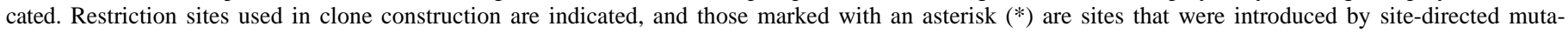

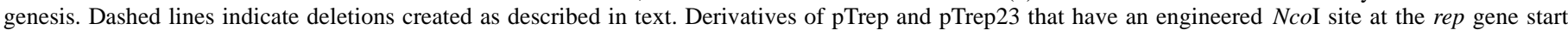
codon were also produced and used in constructs containing DNA-nicking domain mutations as described in text (pTrepN and pTrep23N; and data not shown). 
(27) using primers PRep9-Nco (CATATTTACAACCATGGCACCACCTC) and PAv1885 (CCACTGCGTTGGAATAGAC). Primer PRep9-Nco contains mismatches around the start codon for the rep gene (nucleotides 3 to 6) and changes the sequence from ATATGCC to ccATGgC, thereby creating a NcoI site, which
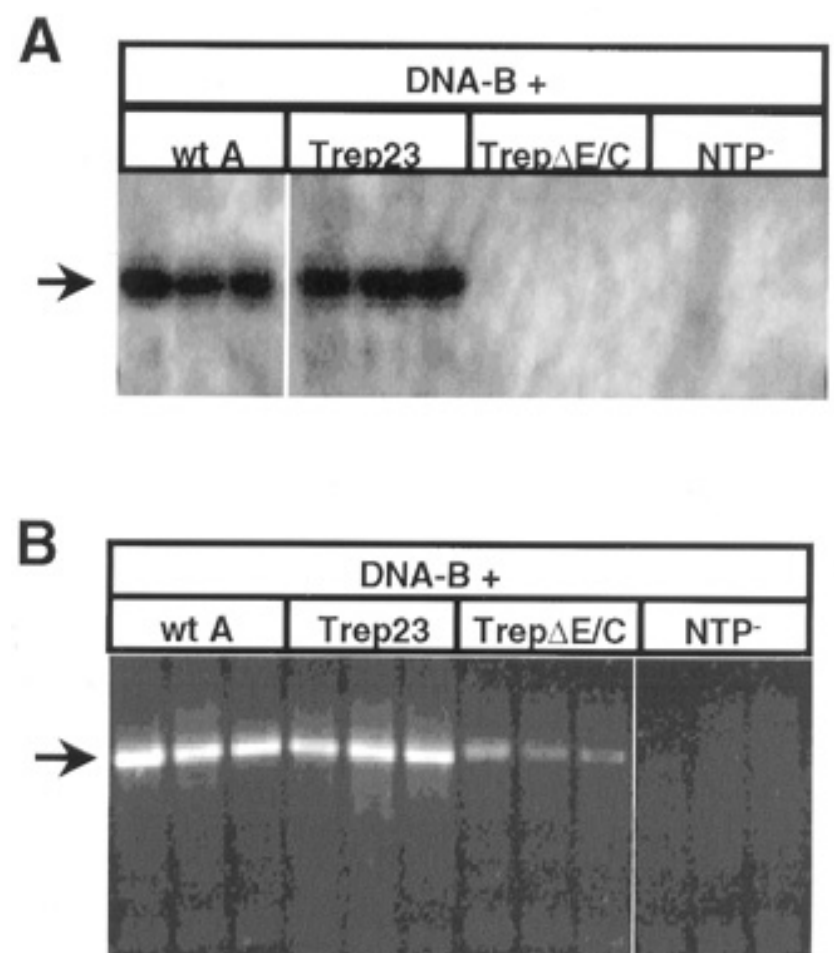

Fig. 2. Replication of bean golden mosaic virus (Guatemalan isolate, BGMVGA) DNA-B following coinoculation into NT-1 tobacco suspension cells with rep gene trans-expression vectors or full-length clones of BGMV-GA DNA-A. NT-1 tobacco suspension cells were coinoculated with DNA-B plus wild-type (wt) BGMV-GA DNA-A (wt A), a BGMV-GA DNA-A clone containing two replication-abolishing nucleoside triphosphate (NTP)-binding domain mutations (E221R and $\mathrm{K} 228 \mathrm{H}, \mathrm{NTP}^{-}$), or trans-expression vectors containing a wt BGMV-GA rep gene (pTrep, pTrep23, or pTrep23 $\Delta \mathrm{E} / \mathrm{C}$ ) as described in text. Total DNA was extracted from cells harvested $72 \mathrm{~h}$ after inoculation. DNA-B replication was detected by A, Southern analysis or $\mathbf{B}$, polymerase chain reaction (PCR). Arrows indicate A, full-length DNA-B replication products or $\mathbf{B}, 1.1-\mathrm{kb}$ PCR products indicative of DNA-B replication. For Southern analysis, $1 \mu \mathrm{g}$ of total DNA was digested with $D p n \mathrm{I}$ and $B g l \mathrm{II}$, resolved on a $1 \%$ agarose gel, blotted onto nylon membrane, and probed with a ${ }^{32} \mathrm{P}$-labeled $c p$ gene probe. Detection of BGMV-GA DNA-B replication products by PCR was performed with $20 \mathrm{ng}$ of $D p n$ I-digested total DNA as previously described (23). matches the engineered NcoI site in pTrepN. The PCR products were digested with $B g l \mathrm{II}$ and $\mathrm{NcoI}$ and cloned into Rep expression vectors in place of the wt $B g l \mathrm{II} / N c o$ I fragment. All clones were descriptively named to indicate the parental vector and the specific rep gene amino acid change mutation encoded, e.g., pTrep23 $\mathrm{Y}^{103} \mathrm{~F}$ is pTrep23N with a mutation that changes codon 103 from $\mathrm{Y}$ to $\mathrm{F}$.

The CaMV 35S promoter was removed from pTrep23N by digestion with $\mathrm{XhoI}$ and partial digestion with $\mathrm{NcoI}$, followed by filling in of the overhangs using T4 DNA polymerase and self-ligation to make pTrep23P-. Expression vectors lacking rep gene start codons were created using site-directed mutagenesis to change the rep gene start codon in pTrep23 and pTrep32 $2 \mathrm{E} / \mathrm{C}$ from ATG to ATC to make pTrep23ATG- and pTrep23 $\Delta$ E/CATG-, respectively.

Analysis of geminiviral DNA replication in NT-1 suspension cells. NT-1 suspension cells were maintained and inoculated by electroporation as previously described (23). All inoculations were performed with a total of $100 \mu \mathrm{g}$ of DNA. All treatments were replicated two or three times within each experiment to control for variability between inoculations. Inoculated NT-1 tobacco suspension cells were incubated at $28^{\circ} \mathrm{C}$ in the dark in all experiments. Cells were harvested $72 \mathrm{~h}$ after inoculation and stored at $-80^{\circ} \mathrm{C}$ until analyzed. Total DNA was extracted from harvested cells as previously described (23).

The ability of rep trans-expression vectors to replicate BGMVGA DNA-B was tested by inoculating NT-1 suspension cells with $5 \mu \mathrm{g}$ each of linearized BGMV-GA DNA-B and $95 \mu \mathrm{g}$ of the indicated rep gene trans-expression plasmid or $20 \mu \mathrm{g}$ of DNA-A plus $75 \mu \mathrm{g}$ of sheared salmon sperm DNA (Sigma Chemical Co., St. Louis). The effect of trans-expressed rep gene on replication of geminiviral DNA-As was determined by coinoculating NT-1 suspension cells with the indicated amounts of DNA-A (1 to $10 \mu \mathrm{g})$ and rep gene trans-expression vectors ( 90 to $99 \mu \mathrm{g}$ ) as indicated.

Replication products were detected by Southern analysis or PCR as previously described (23). For Southern analysis, $1 \mu \mathrm{g}$ of total DNA was digested with 2 units of $D p n I$ overnight at $37^{\circ} \mathrm{C}$ to digest residual inoculum DNA. In most experiments, a second enzyme was also used, which digests the geminiviral DNA at a single site, to convert supercoiled and open circular double-stranded (ds) DNAs into a single band of linear dsDNA to simplify quantification. NcoI was used for BGMV-GA and BGMV-DR DNA-As, while $B g l$ II was used for BGMV-BZ and BDMV DNA-As and for BGMVGA DNA-B. Digested DNAs were resolved on $1 \%$ agarose gels (Seakem LE; FMC BioProducts, Rockland, ME) and blotted onto positively charged nylon membranes (Zeta-bind, Cuno Inc., Meriden, CT; or Qiabrane plus, Qiagen Inc., Chatsworth, CA). ${ }^{32}$ P-labeled probes were produced by random hexamer-labeling of $c p$ gene sequences amplified from cloned DNA-A components by PCR with the degenerate $c p$ gene primers PGVvCP (CTAAGCGYGATGC-

\begin{tabular}{|c|c|c|c|c|c|c|c|c|}
\hline \multirow{2}{*}{\begin{tabular}{|c|} 
DNA-A \\
DTrep23X \\
\end{tabular}} & \multicolumn{7}{|c|}{ wt DNA-A } & \multirow{2}{*}{$\frac{\text { NTP- }}{\mathrm{P}^{-}}$} \\
\hline & P. & ATG- & Y103F & K106R & $\mathrm{K} 228 \mathrm{H}$ & D262R & $\mathrm{DM}$ & \\
\hline Rel. Rep. & 1.00 & 0.96 & 0.01 & 0.01 & 0.05 & 0.01 & 1.01 & 0 \\
\hline Std. Dev. & 0.03 & 0.17 & 0.01 & 0.01 & 0.08 & 0.02 & 0.12 & - \\
\hline
\end{tabular}

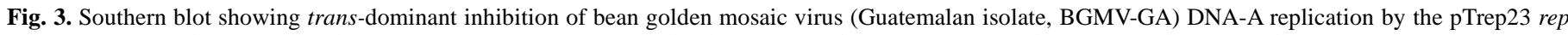

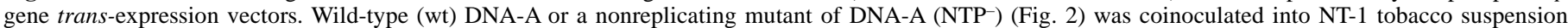

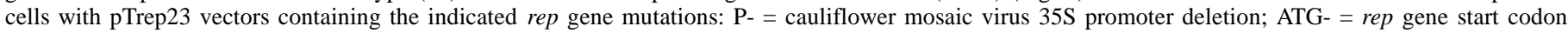

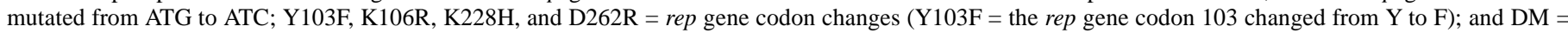

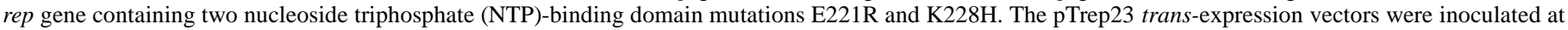

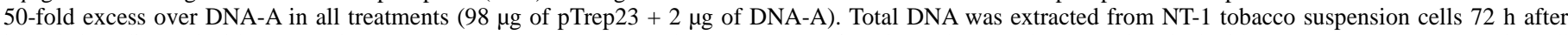

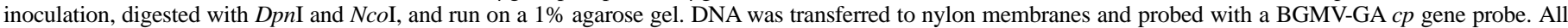

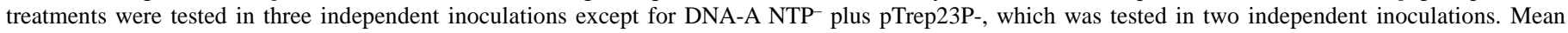

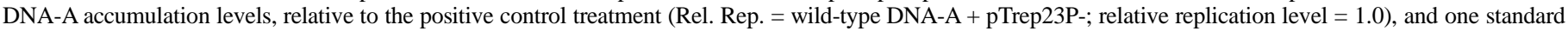
deviation (Std. Dev.) are indicated by numbers below each treatment. 
BCMRTGGCG) and PGVcCP (CGAATRTARAAATAGATCCG). A NcoI fragment corresponding to nucleotides 976 to 1,837 of BGMV-GA DNA-B was used as a probe for DNA-B. Hybridizations were performed in the presence of $30 \%$ formamide as previously described (19). Probed blots were exposed to XAR-5 X-ray film (Eastman Kodak, Rochester, NY) for 2 to $120 \mathrm{~h}$ as necessary to obtain exposures appropriate for quantification. Southern blots were quantified by computer analysis of digitized images using Sigma Scan/Image software (Jandel Scientific Software, San Rafael, CA). A dilution series of linear unit-length, cloned DNA-A was included on each gel as an internal standard for quantification. Data shown represent typical results from experiments that were repeated three or more times.

Molecular biology techniques. All plasmids were purified from Escherichia coli by alkaline lysis and quantified using a fluorometer (TKO-100; Hoefer Scientific Instruments). Restriction endonucleases were obtained from Promega Corp. (Madison, WI) or New England Biolabs, Inc. (Beverly, MA). Molecular biology techniques not described were performed according to established procedures (31).

\section{RESULTS}

Replication of DNA-B by trans-expressed rep genes. transExpression vectors containing a wt BGMV-GA rep gene (Fig. 1) were tested for the ability to replicate BGMV-GA DNA-B to determine if functional Rep protein was produced following inoculation of NT-1 suspension cells with rep gene trans-expression plasmids. Using a PCR-based geminiviral replication assay (23), replication of DNA-B was detected in all treatments in which DNA-B was coinoculated with wt DNA-A or rep gene trans-expression vectors containing a wt rep gene (pTrep23 and pTrep23 $\Delta \mathrm{E} / \mathrm{C}$ ), but not when DNA-B was coinoculated with pGAADM, a BGMV-GA DNA-A clone containing two replication-abolishing rep gene mutations (E221R and $\mathrm{K} 228 \mathrm{H})$, or with rep gene trans-expression vectors containing lethal rep mutations (Fig. 2). When tested by Southern analysis, DNA-B replication was not apparent in treatments inoculated with pTrep $23 \Delta \mathrm{E} / \mathrm{C}$, which indicates that the level of DNA-B replication was substantially reduced relative to treatments in which DNA-B was inoculated with pTrep23 (Fig. 2). These results show that pTrep23 and pTrep23 $\Delta \mathrm{E} / \mathrm{C}$ were able to express functional wt Rep protein and that the full-length $a c 3$ gene contained in pTrep23 enhances the efficiency of replication, while the truncated ac3 gene coded in the pTrep $23 \Delta \mathrm{E} / \mathrm{C}$ vector does not.

trans-Dominant inhibition of BGMV-GA DNA-A replication by trans-expressed rep gene mutants. The ability of trans-expressed rep genes to interfere with geminiviral replication was tested by coinoculating NT-1 suspension cells with wt BGMV-GA and trans-expression plasmids coding for mutant rep genes. transExpression vectors were inoculated in 10- to 100-fold mass excess (13- to 130-fold molar excess) relative to DNA-A as indicated. DNA-A replication was detected by Southern analysis with a probe derived from the $c p$ gene of BGMV-GA. Digestion with DpnI re- moved residual inoculum DNA as previously described (23). Digestion with $N c o$ I, which cuts BGMV-GA DNA-A at a single site at nucleotide 2,117 , in addition to $D p n I$ resulted in detection of linear unit-length dsDNAs in all treatments inoculated with wt BGMV-GA DNA-A (Figs. 2 to 6). BGMV-GA DNA-A replication was easily detected in all treatments in which DNA-A was inoculated with the bacterial cloning vector Bluescript (pBS) (Fig. 2) or Trep23P- (CaMV 35S promoter deletion of pTrep23) (Figs. 3 to 6), which were used as positive controls to establish DNA-A replication levels in the absence of trans-expressed geminiviral genes. No DNA-A replication products were detected in treatments in which NT-1 suspension cells were inoculated with pGAADM, a BGMV-GA DNA-A clone that contains two replication-abolishing mutations in the NTP-binding domain (E221R and K228H) (23). This treatment served as the negative control for DNA-A replication (Figs. 3, 4, and 6). Treatments were replicated two to three times within each experiment, and DNA-A replication levels typically varied less than $20 \%$ between replicated treatments.

BGMV-GA DNA-A accumulation was reduced by 95 to $99 \%$ relative to the positive control treatment when inoculated with rep gene trans-expression vectors coding for most of the nonfunctional rep gene mutants (Fig. 3). The rep gene mutants Y103F, K106R, and D262R were more efficient at inhibiting DNA-A replication than with $\mathrm{K} 228 \mathrm{H}$ (Fig. 3). Interestingly, trans-expression vectors containing the temperature-sensitive rep gene mutation E221R (23), alone or in combination with other rep gene mutations, did not significantly reduce BGMV-GA DNA-A accumulation (Fig. 3, pTrep23DM; and data not shown). rep Gene mutants Y103F and D262R, expressed from the pTrep23 vector, were able to efficiently interfere with DNAA replication when inoculated at only fivefold mass excess, the lowest ratio tested, reducing DNA-A accumulation by 95 to $99 \%$ relative to the positive control treatments (data not shown).

To determine if the full-length trap or $a c 3$ genes present in the pTrep23 vector contributed to trans-dominant activity, the ability of pTrep $23 \Delta \mathrm{E} / \mathrm{C}$ constructs to interfere with BGMV-GA DNA-A replication was tested. The pTrep $23 \Delta \mathrm{E} / \mathrm{C}$ vector is identical to the pTrep23 vectors, except that it contains an EcoRI (nucleotide 1,472) to ClaI (nucleotide 1,299) deletion (Fig. 1), which truncates the trap and $a c 3$ genes and abolishes the replication-stimulating activity of the $a c 3$ gene (Fig. 2). Results from experiments in which DNA-A was coinoculated with pTrep $23 \Delta \mathrm{E} / \mathrm{C}$ vectors containing rep gene mutants showed that full-length trap and $a c 3$ genes were not required for trans-dominant inhibition of geminiviral replication. The DNAnicking domain mutant $\mathrm{Y} 103 \mathrm{~F}$ and the NTP-binding domain mutant D262R were equally efficient at inhibiting BGMV-GA DNA-A replication when expressed from pTrep23 and pTrep23 $\Delta \mathrm{E} / \mathrm{C}$ vectors, reducing DNA-A accumulation by $\sim 95$ to $99 \%$ relative to the positive control treatment when inoculated in 50-fold mass excess over DNA-A (Fig. 4). DNA-A replication was also reduced by 95 to $99 \%$ by $\mathrm{Y} 103 \mathrm{~F}$ and $\mathrm{D} 262 \mathrm{R}$ mutants expressed from pTrep $23 \Delta \mathrm{E} / \mathrm{C}$ when inoculated at ratios of 20:1 and 10:1, respectively, over BGMV-GA DNA-A (Fig. 5; and data not shown).

\begin{tabular}{|l|c|c|c|c|c|}
\hline rep mutation & - & \multicolumn{2}{|c|}{ Y103F } & \multicolumn{2}{c|}{ D262R } \\
\hline pTrepX & pTrep23P. & pTrep23 & pTrep23 $\Delta / C$ & pTrep23 & pTrep23 $\Delta / C$ \\
\hline
\end{tabular}

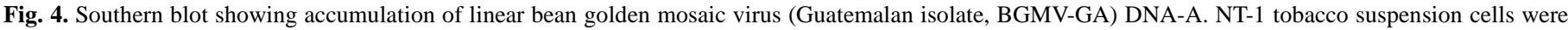

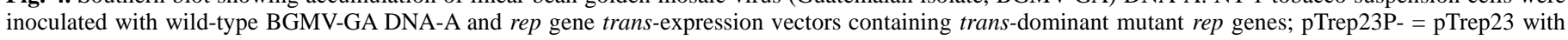

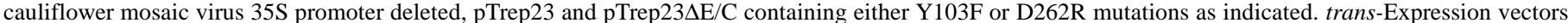

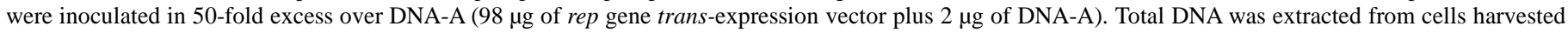

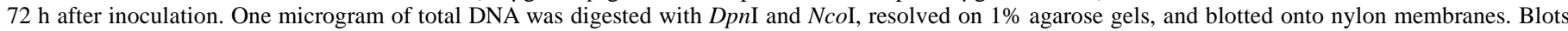
were probed with a ${ }^{32} \mathrm{P}$-labeled BGMV-GA $c p$ gene probe. 
Rep protein mediates trans-dominant inhibition of BGMVGA replication. trans-Dominance appeared to be mediated by the Rep protein, since different rep gene mutants inhibited DNA-A replication to different degrees $(\mathrm{Y} 103 \mathrm{~F}=\mathrm{D} 262 \mathrm{R}>\mathrm{K} 228 \mathrm{H})$, and because rep genes containing the temperature-sensitive E221R mutation were not trans-dominant (Fig. 3). The rep gene expression vector pTrep23ATG-, which contains a mutation that changes the rep gene start codon from ATG to ATC, was used to definitively establish that Rep protein was necessary for trans-dominance and to assess whether RNA-mediated mechanisms such as cosuppression (9) contributed to trans-dominance. No reduction of DNA-A accumulation was observed when NT-1 suspension cells were coinoculated with DNA-A and pTrep23ATG- (Fig. 3), indicating that Rep protein is required for trans-dominance and that RNA-mediated mechanisms such as cosuppression (9) do not contribute significantly to trans-dominance in this system.

Inhibition of heterologous geminiviral replication. We tested the ability of the DNA-nicking domain mutant Y103F and the NTPbinding domain mutant D262R to inhibit replication of DNA-As from BGMV-DR (18), BGMV-BZ (18), and BDMV (25). Infectious clones of DNA-A for each virus were coinoculated with a 50-fold excess of the indicated rep gene trans-expression vector, and replication was detected by Southern analysis using a $c p$ gene probe for each virus. Each DNA-A for these viruses replicated well and accumulated dsDNA replication products when inoculated with the promoterless rep gene trans-expression plasmid pTrep23P- (Fig. 6). Interestingly, replication of DNA-As from all three geminiviruses was inhibited by the DNA-nicking domain mutant Y103F and the NTP-binding domain mutant D262R when expressed from pTrep23 $\mathrm{E} / \mathrm{C}$ (Fig. 6). These results show that trans-dominant rep genes can interfere with the replication of heterologous geminiviruses as well as the geminivirus from which the trans-dominant rep gene was derived.

\section{DISCUSSION}

The lack of natural sources of resistance sufficient for control of diseases caused by geminiviruses has led to the exploration of engineered resistance strategies (17). We chose to target the rep gene for engineering resistance, since it is the only geminiviral gene necessary for DNA replication $(22,23)$. We previously reported genetic studies that demonstrated that the putative DNA-nicking and NTP-binding domains, which are conserved in the rep gene of every geminivirus, are necessary for geminiviral DNA replication $(23,27)$. Here we report experiments testing the ability of rep genes containing lethal mutations within these domains to function as trans-dominant inhibitors of geminiviral replication.

The ability of mutant rep genes to trans-dominantly inhibit geminiviral replication was tested using a transient assay in which rep

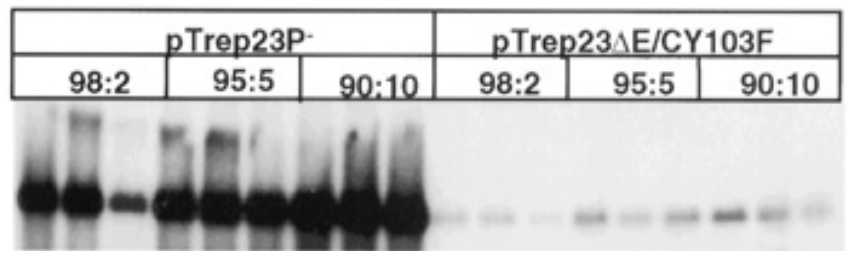

Fig. 5. Southern blot of trans-dominant inhibition of bean golden mosaic virus (Guatemalan isolate, BGMV-GA) DNA-A replication by pTrep $\Delta \mathrm{E} / \mathrm{CY} 103 \mathrm{~F}$ at different inoculation ratios (98:2, 95:5, and 90:10). NT-1 tobacco suspension cells were coinoculated with BGMV-GA DNA-A and pTrep23 2 E/CY103F or pTrep23P- (promoterless rep gene trans-expression vector) (Fig. 1) at the indicated ratio. Three independent inoculations were performed for each treatment. Total DNA was extracted from cells harvested at $72 \mathrm{~h}$ after inoculation. One microgram of total DNA was digested with DpnI and NcoI, resolved on a $1 \%$ agarose gel, and blotted onto nylon membrane. The blot was probed with a ${ }^{32} \mathrm{P}-$ labeled BGMV-GA $c p$ gene probe. The blot was exposed for 5 days so that DNA-A replication levels could be determined in all treatments. Quantification of DNA-A accumulation indicated that DNA-A replication was reduced by $\sim 99 \%$ in all treatments containing pTrep $23 \Delta \mathrm{E} / \mathrm{CY} 103 \mathrm{~F}$. gene expression plasmids were coinoculated with cloned geminiviral DNA-As into NT-1 suspension cells. This transient assay was developed to allowed rapid screening of many potential transdominant mutants, independent of difficult-to-control variables such as position and copy number effects, prior to construction of transgenic plants (20).

Our results show that the wt rep gene from BGMV-GA is necessary for replication of BGMV-GA DNA-B in NT-1 tobacco suspension cells and that the $a c 3$ gene enhances replication. These data indicate that functional Rep and Ac3 proteins are produced after inoculation of NT-1 suspension cells with the rep gene transexpression vectors used and are consistent with a number of previous studies characterizing the roles of rep and $a c 3$ genes in geminiviral DNA replication $(23,27,44)$.

We found that trans-expression of several nonfunctional mutant rep genes significantly inhibited replication of BGMV-GA DNA-A. The lack of trans-dominant activity in constructs lacking a start codon for the rep gene demonstrates that the Rep protein is required for trans-dominance and that the full-length or truncated trap and ac 3 genes encoded by $\mathrm{pTrep} 23$ and $\mathrm{pTrep} 23 \Delta \mathrm{E} / \mathrm{C}$ vectors are not sufficient for trans-dominant inhibition of geminivirus replication. These results also showed that RNA-mediated mechanisms such as cosuppression (9) did not contribute to trans-dominant activity.

The E221R mutation in the rep gene of BGMV-GA was previously shown to have a temperature-sensitive phenotype for replication (23) and was the only rep gene mutant that was not a transdominant inhibitor of geminiviral replication. Interestingly, the E221R mutation suppressed the trans-dominant phenotype of other rep gene mutants such as $\mathrm{K} 228 \mathrm{H}$ (pTrep23DM). All experiments reported here were performed at $28^{\circ} \mathrm{C}$, a nonpermissive temperature for replication mediated by rep genes containing the E221R mutation (23). The E221R mutation probably causes a conformational change at the nonpermissive temperature that reduces the stability of the Rep protein or alters the tertiary structure of the Rep protein such that it can no longer participate in replication or trans-dominance. Despite intensive efforts, we have not been able to quantify Rep protein in transfected NT-1 tobacco culture cells to test this hypothesis.

Taken together, these results show that trans-dominant inhibition of geminiviral replication requires, and may be mediated directly by, nonfunctional Rep protein. Though not sufficient for trans-domi-

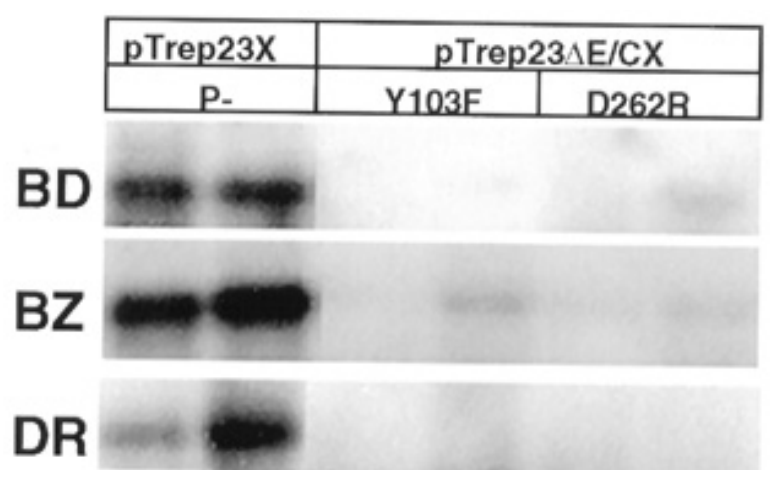

Fig. 6. Southern blots of geminiviral DNA-A replication in the presence of rep gene trans-expressed mutants of bean golden mosaic virus (Guatemalan isolate, BGMV-GA). DNA-As from Dominican Republic (-DR) or Brazilian isolates (-BZ) of BGMV or bean dwarf mosaic virus (BDMV) were coinoculated into NT-1 tobacco suspension cells with the indicated rep gene transexpression vector at a ratio of 50:1 (98 $\mu \mathrm{g}$ of rep gene trans-expression vector plus $2 \mu \mathrm{g}$ of DNA-A). Total DNA was extracted from cells harvested $72 \mathrm{~h}$ after inoculation. One microgram of total DNA was digested with either DpnI and $N c o I$ (BGMV-DR treatments) or DpnI and BglII (BGMV-BZ and BDMV treatments). Digested DNAs were resolved on $1 \%$ agarose gels, blotted onto nylon membranes, and probed with ${ }^{32} \mathrm{P}$-labeled $c p$ gene probe from the corresponding geminivirus. Two independent inoculations were performed for each treatment. Panels show DNA-A replication products for BDMV (BD), BGMV-BZ (BZ), and BGMV-DR (DR). 
nance, it is possible that the full-length and truncated trap and ac3 genes and associated downstream sequences contribute to transdominance. It is interesting that sequences containing either full-length or nonfunctional truncated $a c 3$ open reading frames were equally efficient at enhancing the activity of trans-dominant rep genes.

We tested the ability of mutant rep genes derived from BGMVGA to interfere with the replication of several heterologous geminiviruses, which also cause severe losses on beans in Latin America including BGMV-DR, BGMV-BZ, and BDMV. BGMV-GA and BGMV-DR are very closely related, since the DNA-As are $\sim 97 \%$ identical at the sequence level, the direct repeat sequences in the ori that are bound by the Rep protein are identical, and BGMVGA and BGMV-DR can infect plants as pseudorecombinants comprised of DNA-A from one virus and DNA-B of the other (13). BGMV-GA is not closely related to BGMV-BZ or BDMV, both of which are only about $75 \%$ identical to BGMV-GA at the sequence level and unable to form pseudorecombinants with BGMV-GA (13). Interestingly, mutant rep genes derived from BGMV-GA inhibited replication of all three geminiviral DNA-As. This observation indicates that trans-dominance is at least partially mediated by a mechanism that is independent of Rep protein-ori DNA interactions, since the Rep protein of BGMV-GA does not bind to the ori sequences of BGMV-BZ or BDMV under conditions in which binding to ori sequences of BGMV-GA is easily detectable (S. F. Hanson, unpublished data).

The data presented here complement and extend several other studies examining the ability of trans-expressed rep genes to interfere with geminiviral replication. Truncated rep genes from African cassava mosaic virus (ACMV) and TYLCV-Sar have been shown to interfere with geminiviral replication in transgenic $N$. benthamiana plants $(26,35)$. Though no immune lines were discovered, several lines with high levels of resistance were identified in each experiment. In both cases, the truncated rep gene mutants, which interfered with replication, coded for Rep proteins that contain the ori DNA-binding domain but lacked the NTP-binding domain. Plants with resistance to ACMV were completely susceptible to TGMV and beet curly top geminivirus, indicating that the resistance had a narrow spectrum $(26,35)$. The TYLCV-Sar-resistant plants were not tested for resistance to heterologous geminiviruses (35). The constructs used to engineer the ACMV-resistant plants did not contain sequences downstream of the rep gene analogous to those included in pTrep23 and pTrep23 $\Delta \mathrm{E} / \mathrm{C}$. Thus, it is possible that the ac3 sequences used in our experiments contributed to the broad-spectrum trans-dominant activity we observed.

The conservation of the DNA-nicking and NTP-binding domains in all geminivirus rep genes indicates that this approach may be useful in generating resistance genes to any geminivirus. In addition to the results reported here, we have found that similar rep gene mutants from BGMV-BZ are trans-dominant inhibitors of BGMVBZ replication in the NT-1 tobacco suspension cell system (J. C. Faria and D. P. Maxwell, personal communication). We have also produced transgenic tomatoes that express trans-dominant ToMoV rep gene mutants that are resistant to ToMoV when challenged by inoculation with the natural insect vector, Bemisia tabaci (41).

Generation of broad-spectrum resistance to geminiviruses is an important goal, since the known diversity of geminiviruses on any given crop may be a limiting factor in successful application of narrow-spectrum resistance genes (e.g., tomatoes in Central America [21]). The results presented here suggest that broad-spectrum resistance may be attained using trans-dominant rep gene mutants and associated downstream sequences. Experiments are in progress to determine if the results obtained with the transient assay system can be duplicated in transgenic plants.

\section{ACKNOWLEDGMENTS}

This research was supported, in part, by the College of Agricultural and Life Sciences, by a grant from USAID as part of the Bean/Cowpea
CRSP, by USDA grant no. 9101351, and by Hatch funds. We thank M. D. Maxwell for critical review of the manuscript and P. Ahlquist and R. L. Gilbertson for helpful discussions.

\section{LITERATURE CITED}

1. Abouzid, A. M., Polston, J. E., and Hiebert, E. 1992. The nucleotide sequence of tomato mottle virus, a new geminivirus isolated from tomatoes in Florida. J. Gen. Virol. 73:3225-3229.

2. Argüello-Astorga, G. R., Guevara-González, R. G., Herrera-Estrella, L. R., and Rivera-Bustamante, R. F. 1994. Geminivirus replication origins have a group-specific organization of iterative elements: A model for replication. Virology 203:90-100.

3. Beebe, S. E., Ochoa, I., Skroch, P., and Tivang, J. 1995. Genetic diversity among common bean breeding lines developed for Central America. Crop Sci. 35:1178-1183.

4. Blair, M. W., Bassett, M. J., Abouzid, A. M., Hiebert, E., Polston, J. E., McMillan, Jr., R. T., Graves, W., and Lamberts, M. 1995. Occurrence of bean golden mosaic virus in Florida. Plant Dis. 79:529-533.

5. Blair, M. W., and Beaver, J. S. 1993. Inheritance of bean golden mosaic virus resistance from bean genotype A429. Annu. Rep. Bean Improv. Coop. 36:143.

6. Brown, J. K. 1994. The present status of Bemisia tabaci as a plant pest and virus vector in agroecosystems world wide. FAO Plant Prot. Bull. 42:3-31.

7. Brown, J. K., and Bird, J. 1992. Whitefly-transmitted geminiviruses and associated disorders in the Americas and the Caribbean Basin. Plant Dis. 76:220-225.

8. Desbiez, C., David, C., Mettouchi, A., Laufs, J., and Gronenborn, B. 1995. Rep protein of tomato yellow leaf curl geminivirus has an ATPase activity required for viral DNA replication. Proc. Natl. Acad. Sci. U.S.A. 92: 5640-5644.

9. Dougherty, W. G., and Parks, T. D. 1995. Transgenes and gene suppression: Telling us something new? Curr. Opin. Cell Biol. 7:399-405.

10. Eagle, P. A., Orozco, B. M., and Hanley-Bowdoin, L. 1994. A DNA sequence required for geminivirus replication also mediates transcriptional regulation. Plant Cell 6:1157-1170.

11. Elmer, J. S., Brand, L., Sunter, G., Gardiner, W. E., Bisaro, D. M., and Rogers, S. G. 1988. Genetic analysis of the tomato golden mosaic virus II. The product of the AL1 coding sequence is required for replication. Nucleic Acids Res. 16:7043-7060.

12. Etessami, P., Saunders, K., Watts, J., and Stanley, J. 1991. Mutational analysis of complementary-sense genes of African cassava mosaic virus DNA A. J. Gen. Virol. 72:1005-1012.

13. Faria, J. C., Gilbertson, R. L., Hanson, S. F., Morales, F. J., Ahlquist, P., Loniello, A. O., and Maxwell, D. P. 1994. Bean golden mosaic geminivirus type II isolates from the Dominican Republic and Guatemala: Nucleotide sequences, infectious pseudorecombinants, and phylogenetic relationships. Phytopathology 84:321-329.

14. Fontes, E. P. B., Eagle, P. A., Sipe, P. S., Lucknow, V. A., and HanleyBowdoin, L. 1994. Interaction between a geminivirus replication protein and origin DNA is essential for viral replication. J. Biol. Chem. 269: 8459-8465.

15. Fontes, E. P. B., Gladfelter, H. J., Schaffer, R. L., Petty, I. T. D., and Hanley-Bowdoin, L. 1994. Geminivirus replication origins have a modular organization. Plant Cell 6:405-416.

16. Fontes, E. P. B., Luckow, V. A., and Hanley-Bowdoin, L. 1992. A geminivirus replication protein is a sequence-specific DNA binding protein. Plant Cell 4:597-608.

17. Frischmuth, T., and Stanley, J. 1993. Strategies for the control of geminivirus diseases. Semin. Virol. 4:329-337.

18. Gilbertson, R. L., Faria, J. C., Hanson, S. F., Morales, F. J., Ahlquist, P., Maxwell, D. P., and Russell, D. R. 1991. Cloning of the complete DNA genomes of four bean-infecting geminiviruses and determining their infectivity by electric discharge particle acceleration. Phytopathology 81: 980-985.

19. Gilbertson, R. L., Hidayat, S. H., Martinez, R. T., Leong, S. A., Faria, J. C., Morales, F., and Maxwell, D. P. 1991. Differentiation of bean-infecting geminiviruses by nucleic acid hybridization probes and aspects of bean golden mosaic in Brazil. Plant Dis. 75:336-342.

20. Goodwin, J., Chapman, K., Swaney, S., Parks, T. D., Wernsman, E. A., and Dougherty, W. G. 1996. Genetic and biochemical dissection of transgenic RNA-mediated virus resistance. Plant Cell 8:95-105.

21. Green, S. K., and Kallo, G. 1994. Leaf curl and yellowing viruses of pepper and tomato: An overview. Asian Veg. Res. Dev. Cent. Tech. Bull. 21: $1-51$.

22. Hanley-Bowdoin, L., Elmer, J. S., and Rogers, S. G. 1990. Expression of functional replication protein from tomato golden mosaic virus in transgenic tobacco plants. Proc. Natl. Acad. Sci. U.S.A. 87:1446-1450.

23. Hanson, S. F., Hoogstraten, R. A., Ahlquist, P., Gilbertson, R. L., Russell, 
D. R., and Maxwell, D. P. 1995. Mutational analysis of a putative NTPbinding domain in the replication-associated protein (AC1) of bean golden mosaic geminivirus. Virology 211:1-9.

24. Heibert, E., Abouzid, A. M., and Polston, J. E. 1996. Whitefly-transmitted geminiviruses. Pages 277-288 in: Bemisia 1995: Taxonomy, Biology, Damage, Control and Management. D. Gerling and R. T. Mayer, eds. Intercept Ltd., Andover, United Kingdom.

25. Hidayat, S. H., Gilbertson, R. L., Hanson, S. F., Morales, F. J., Ahlquist, P., Russell, D. R., and Maxwell, D. P. 1993. Complete nucleotide sequences of the infectious cloned DNAs of bean dwarf mosaic geminivirus. Phytopathology 83:181-187.

26. Hong, Y., and Stanley, J. 1996. Virus resistance in Nicotiana benthamiana conferred by African cassava mosaic virus replication-associated protein (AC1) transgene. Mol. Plant-Microbe Interact. 9:219-225.

27. Hoogstraten, R. A., Hanson, S. F., and Maxwell, D. P. 1996. Mutational analysis of the putative nicking motif in the replication-associated protein $(\mathrm{AC} 1)$ of bean golden mosaic geminivirus. Mol. Plant-Microbe Interact. 9:594-599.

28. Laufs, J., Jupin, I., David, C., Schumacher, S., Heyraud, F., and Gronenborn, B. 1995. Geminivirus replication: Genetic and biochemical characterization of Rep protein function, a review. Biochimie 77:765-773.

29. Laufs, J., Traut, W., Heyraud, F., Matzeit, V., Rogers, S. G., Schell, J., and Gronenborn, B. 1995. In vitro cleavage and joining at the viral origin of replication by the replication initiator protein of tomato yellow leaf curl virus. Proc. Natl. Acad. Sci. U.S.A. 92:3879-3883.

30. Li, X., and Rhode, III, S. L. 1990. Mutation of lysine 405 to serine in the parvovirus $\mathrm{H}-1$ NS1 abolishes its functions for viral DNA replication, late promoter trans activation, and cytotoxicity. J. Virol. 64:4654-4660.

31. Maniatis, T., Fritsch, E. F., and Sambrook, J. 1989. Molecular Cloning: A Laboratory Manual. 2nd ed. Cold Spring Harbor Press, Cold Spring Harbor, NY.

32. Morales, F. J., and Singh, S. P. 1991. Genetics of resistance to bean golden mosaic virus in Phaseolus vulgaris L. Euphytica 52:113-118.

33. Morris, B., Richardson, K., Eddy, P., Zhan, X., Haley, A., and Gardner, R. 1991. Mutagenesis of the AC3 open reading frame of African cassava mosaic virus DNA A reduces DNA B replication and ameliorates disease symptoms. J. Gen. Virol. 72:1205-1214.

34. Nateshan, H. M., Myniyappa, V., Jalikop, S. H., and Mamappa, H. K. 1996. Resistance of Lycopersicon species and hybrids to tomato leaf curl geminivirus. Pages 369-377 in: Bemisia 1995: Taxonomy, Biology, Damage, Control and Management. D. Gerling and R. T. Mayer, eds. Intercept Ltd., Andover, United Kingdom.

35. Noris, E., Accotto, G. P., Tavazza, R., Brunetti, A., Crespi, S., and Tavazza, M. 1996. Resistance to tomato yellow leaf curl geminivirus in Nicotiana benthamiana plants transformed with a truncated viral $\mathrm{C} 1$ gene. Virology
224:130-138.

36. Pedersen, T. J., and Hanley-Bowdoin, L. 1994. Molecular characterization of the AL3 protein encoded by a bipartite geminivirus. Virology 202: 1070-1075.

37. Saunders, K., Lucy, A., and Stanley, J. 1991. DNA forms of the geminivirus African cassava mosaic virus consistent with a rolling circle mechanism of replication. Nucleic Acids Res. 19:2325-2330.

38. Smith, C. A., and Deluca, N. A. 1992. Transdominant inhibition of herpes simplex virus growth in transgenic mice. Virology 191:581-588.

39. Stanley, J. 1995. Analysis of African cassava mosaic virus recombinants suggests strand nicking occurs within the conserved nonanucleotide motif during the initiation of rolling circle DNA replication. Virology 206: 707-712

40. Stenger, D. C., Revington, G. N., Stevenson, M. C., and Bisaro, D. M. 1991. Replicational release of geminivirus genomes from tandemly repeated copies: Evidence for rolling-circle replication of a plant viral DNA. Proc. Natl. Acad. Sci. U.S.A. 88:8029-8033.

41. Stout, J. T., Liu, H. T., Polston, J. E., Gilbertson, R. L., Nakhla, M. K., Hanson, S. F., and Maxwell, D. P. 1997. Engineered rep gene-mediated resistance to tomato mottle geminivirus in tomato. (Abstr.) Phytopathology 87:S94.

42. Stow, N. D., Hammarsten, O., Arbuckle, M. I., and Elias, P. 1993. Inhibition of herpes simplex virus type 1 DNA replication by mutant forms of the origin-binding protein. Virology 196:413-418.

43. Sunter, G., Hartitz, M. D., and Bisaro, D. M. 1993. Tomato golden mosaic virus leftward gene expression: Autoregulation of geminivirus replication protein. Virology 195:275-280.

44. Sunter, G., Hartitz, M. D., Hormuzdi, S. G., Brough, C. L., and Bisaro, D. M. 1990. Genetic analysis of tomato golden mosaic virus: ORF AL2 is required for coat protein accumulation while ORF AL3 is necessary for efficient DNA replication. Virology 179:69-77.

45. Timmermans, M. C. P., Das, O. P., and Messing, J. 1994. Geminiviruses and their uses as extrachromosomal replicons. Annu. Rev. Plant Physiol. Plant Mol. Biol. 45:79-112.

46. Van Mansfeld, A. D. M., van Teeffelen, H. A. A. M., Baas, P. D., and Jansz, H. S. 1986. Two juxtaposed tryrosyl-OH groups participate in $\Phi X-174$ gene A protein catalysed cleavage and ligation of DNA. Nucleic Acids Res. 14:4229-4238

47. Vidavsky, F., and Czosnek, H. 1998. Tomato breeding lines resistant and tolerant to tomato yellow leaf curl virus issued from Lycopersicon hirsutum. Phytopathology 88:910-914.

48. Zakay, Y., Navot, N., Zeidan, M., Kedar, N., Rabinowitch, H., Czosnek, H., and Zamir, D. 1991. Screening Lycopersicon accessions for resistance to tomato yellow leaf curl virus: Presence of viral DNA and symptom development. Plant Dis. 75:279-281. 